\title{
What are zeta functions of graphs and what are they good for?
}

\author{
Matthew D. Horton, H. M. Stark, and Audrey A. Terras
}

\begin{abstract}
We discuss zeta functions of finite irregular undirected connected graphs (which may be weighted) and apply them to obtain, for example an analog of the prime number theorem for cycles in graphs. We consider 3 types of zeta functions of graphs: vertex, edge, and path. Analogs of the Riemann hypothesis are also introduced.
\end{abstract}

\section{Introduction}

First let us tell some zeta stories.

The Riemann zeta function is defined for $\operatorname{Re}(s)>1$ by

$$
\zeta(s)=\sum_{n=1}^{\infty} \frac{1}{n^{s}}=\prod_{p=\text { prime }}\left(1-p^{-s}\right)^{-1} .
$$

In 1859 Riemann extended this function to all complex numbers $s$ as a meromorphic function with its only pole (a simple one) at $s=1$. He also showed that there is a functional equation relating $\zeta(s)$ and $\zeta(1-s)$. The functional equation involves the gamma function and says $\Lambda(s)=\pi^{-s / 2} \Gamma\left(\frac{s}{2}\right) \zeta(s)=\Lambda(1-s)$.

Riemann's main interest in zeta was as a means to prove the prime number theorem (proved by Hadamard and de la Vallée Poussin in 1896) which says

$$
\#\{p \quad \mid p=\text { prime }, p \leq x\} \sim \frac{x}{\log x}, \text { as } \quad x \rightarrow \infty .
$$

The Hadamard product for $s(s-1) \Lambda(s)$ gives a relation between the primes and the zeros of zeta. There are always zeros at the negative even integers (the trivial zeros). The still unproved Riemann hypothesis, which we will call the RH, says the non-trivial zeros of $\zeta(s)$ are on the line $\operatorname{Re}(s)=\frac{1}{2}$. It has been checked for a ridiculously large number of zeros. The $\mathrm{RH}$ has implications for the error term in the prime number theorem. A reference containing a translation of Riemann's paper is Edwards [7]. Recently it has been noticed that (assuming the RH) the spacings of the imaginary parts of the non-trivial zeros of $\zeta(s)$ behave like the eigenvalues of random Hermitian matrices. This relates zeta and quantum chaos. Katz and Sarnak [16] call this the Montgomery-Odlyzko law (another name is GUE for Gaussian Unitary Ensemble) although the law does not appear to have found a proof. 
Many other kinds of zeta functions have been investigated since Riemann. In number theory there is the Dedekind zeta function of an algebraic number field $K$, such as $\mathbb{Q}(\sqrt{3})$, for example. This zeta is an infinite product over prime ideals $\mathfrak{p}$ in $O_{K}$, the ring of algebraic integers of $K$. The terms in the product are $\left(1-N \mathfrak{p}^{-s}\right)^{-1}$, where $N \mathfrak{p}=\#\left(O_{K} / \mathfrak{p}\right)$. Riemann's work can be extended to this function and it can be used to prove the prime ideal theorem. The $\mathrm{RH}$ is unproved but conjectured to be true for the Dedekind zeta function, except for some modifications concerning a possible zero near 1 on the real axis called a "Siegel zero." A reference for this zeta is Lang [19]. There are also function field zeta functions where $K$ is replaced by a finite algebraic extension of $\mathbb{F}_{q}(x)$, the rational functions of one variable over a finite field $\mathbb{F}_{q}$. The RH has been proved for this zeta which is a rational function of $u=q^{-s}$. See Rosen [24]. Moreover, the Montgomery-Odlyzko behavior has been proved for function fields of generic curves as $q$ and the genus approach infinity. See Katz and Sarnak [16], [17].

Selberg defined a zeta function in 1956 (see [26]) that is associated to a compact Riemannian manifold $M=\Gamma \backslash H$, where $H$ is the Poincaré upper half plane with the metric $d s^{2}=y^{-2}\left(d x^{2}+d y^{2}\right)$. Here $\Gamma$ is a discrete group of real fractional linear transformations of positive determinant. The "primes" in this case are primitive closed geodesics $P$ in $M$ of Poincaré length $\nu(P)$. The Selberg zeta function of $M$ is

$$
Z(s)=\prod_{[P]} \prod_{j=0}^{\infty}\left(1-e^{-(s+j) \nu(P)}\right) .
$$

In fact, $Z(s+1) / Z(s)$ is more like the Riemann zeta function. References are Elstrodt [8], Hejhal [11], and Terras [32]. The Selberg trace formula gives a relation between the primes of $M$ and the eigenvalues of the Poincare Laplacian $\Delta=y^{s}\left(\frac{\partial^{2}}{\partial x^{2}}+\frac{\partial^{2}}{\partial y^{2}}\right)$ on $M$. For compact $M$, the Selberg zeta function satisfies the analog of the $\mathrm{RH}$.

Finally there is the Ruelle zeta function of a dynamical system. See Ruelle [25] or Bedford, Keane, and Series [2].

The graph theory zetas first appeared in work of Ihara on p-adic groups in the 1960s. Soon the connection with graphs was found and many papers appeared. The main authors in the 1980s and 90s were Sunada, Hashimoto [10], and Bass [1]. Other references are Venkov and Nikitin [33] and Northshield's paper in the volume of Hejhal et al [12] See [31] for more history and references. The main properties of the Riemann zeta function have graph theory analogs, at least for regular graphs. For irregular graphs there is no known functional equation and it is difficult to formulate the Riemann hypothesis, but we will try in Section 2. In Sections 3 and 4 we will consider the multivariable zeta functions known as edge and path zeta functions of graphs. We will show how to specialize the path zeta to the edge zeta and the edge zeta to the original Ihara (vertex) zeta.

Here we attempt to survey some of the basic theory as presented in Stark and Terras $[\mathbf{2 8}],[\mathbf{2 9}],[\mathbf{3 0}]$. We include the graph theory prime number theorem and our version of Bass's proof of the determinant formula. We will omit the Artin Lfunctions of Galois graph coverings which are developed in [29], for want of space. We will show how to modify the definitions to obtain zeta functions of weighted graphs. We will show what happens to the edge zeta if you delete an edge (fission) and what happens to the path zeta if you collapse an edge to a point (fusion). 
We do not consider zeta functions of infinite graphs in this paper. Such zeta functions are considered, for example, by Clair and Mokhtari-Sharghi [5] as well as Grigorchuk and Zuk [9].

\section{Vertex Zeta Functions}

First we need to find out what primes in graphs are.

We will always assume that our graphs $\mathrm{X}$ are finite, connected, rank $\geq \mathbf{1}$ with no danglers (i.e., degree 1 vertices). Here "rank" means the rank of the fundamental group of the graph (given more explicitly in the next paragraph). Let us recall some of the definitions basic to Stark and Terras [28], [29], [30].

If $X$ is any connected finite undirected graph with vertex set $V$ and (undirected) edge set $E$, we orient its edges arbitrarily and obtain $2|E|$ oriented edges labelled

$$
e_{1}, e_{2}, \cdots, e_{n}, e_{n+1}=e_{1}^{-1}, \ldots, e_{2 n}=e_{n}^{-1} .
$$

"Primes" $[P]$ in $X$ are equivalence classes of closed backtrackless tailless primitive paths $P$. Write a path $C=a_{1} a_{2} \cdots a_{s}$, where $a_{j}$ is an oriented edge of $X$. The length of $C$ is $\nu(C)=s$. Backtrackless means that $a_{i+1} \neq a_{i}^{-1}$, for all $i$. Tailless means that $a_{s} \neq a_{1}^{-1}$. The equivalence class $[C]$ is the set

$$
[C]=\left\{a_{1} a_{2} \cdots a_{s}, a_{2} a_{3} \cdots a_{s} a_{1}, \ldots, a_{s} a_{1} \cdots a_{s-1}\right\} .
$$

$[P]$ is primitive means $P \neq D^{m}$, for any integer $m \geq 2$ and path $D$ in $X$. Here $r_{X}$ will denote the rank of the fundamental group of $X$. We have $r_{X}-1=|E|-|V|$. Then $r_{X}$ is the number of edges deleted from $X$ to form a spanning tree.

The Ihara (vertex) zeta function of $X$ is defined at $u \in \mathbb{C}$, for $|u|$ sufficiently small, by

$$
\zeta(u, X)=\zeta_{V}(u, X)=\prod_{[P]}\left(1-u^{\nu(P)}\right)^{-1},
$$

where $[P]$ runs over the primes of $X$. Here $V$ is for vertex. In this section, we will drop $V$ most of the time for the vertex zeta. In later sections we will define two other kinds of zeta associated to a graph .

The generalization of Ihara's formula (proved by Hashimoto and Bass) says

$$
\zeta(u, X)^{-1}=\left(1-u^{2}\right)^{r_{X}-1} \operatorname{det}\left(I-A_{X} u+Q_{X} u^{2}\right),
$$

where $r_{X}$ is the rank of the fundamental group of $X, A_{X}$ is the adjacency matrix of $X, Q_{X}$ is the diagonal matrix whose jth diagonal entry is $(-1+$ degree of $j$ th vertex). We will give a version of Bass's proof of Ihara's formula in the next section. There are many other proofs. See Stark and Terras [28] for one such. In Terras [31] there is a proof for k-regular graphs using the Selberg trace formula on the k-regular tree.

Definition 2.1. $R_{X}$ is the radius of the largest circle of convergence of the Ihara zeta function.

When $X$ is a $(q+1)$-regular graph, $R_{X}=1 / q$. We will say more about the size of $R_{X}$ for irregular graphs later in this section. As with the Dedekind zeta function, $\zeta(u, X)$ has a meromorphic continuation to the entire complex $u$ plane, but now $\zeta(u, X)^{-1}$ is entire. Thus if we want to formulate a version of the $\mathrm{RH}=$ Riemann Hypothesis, we must consider the poles of $\zeta(u, X)$, not the zeroes. 
In general, $\zeta(u, X)^{-1}$ is a polynomial of degree $2 m$, where $m=|E|$, the number of edges of $X$.

Definition 2.2. We define the $\mathbf{0 , 1}$ edge matrix $W_{1}$ by orienting the $m$ edges of $X$ and labeling them as in formula (2.1). Then $W_{1}$ is the $2 m \times 2 m$ matrix with $i j$ entry 1 if edge $e_{i}$ feeds into $e_{j}$ provided that $e_{j} \neq e_{i}^{-1}$, and $i j$ entry 0 otherwise. By " $a$ feeds into $b$," we mean that the terminal vertex of edge $a$ is the same as the initial vertex of edge $b$.

We will show in the next section (see Corollary 1 ) that

$$
\zeta(u, X)^{-1}=\operatorname{det}\left(I-W_{1} u\right) .
$$

From this we shall derive Ihara's formula (2.3).

Definition 2.3. For a graph $X$ with $m$ edges which have weights given by a weighting function $L: E \rightarrow \mathbb{R}^{+}$, define the weighted length of a closed path $C=a_{1} a_{2} \cdots a_{s}$, where $a_{j}$ is an oriented edge of $X$, by

$$
\nu(C, L)=\nu_{X}(C, L)=\sum_{i=1}^{s} L\left(a_{i}\right) .
$$

Definition 2.4. The Ihara zeta function of a weighted (undirected) graph for $|u|$ small and $u \notin(-\infty, 0)$ is

$$
\zeta(u, X, L)=\prod_{[P]}\left(1-u^{\nu(P, L)}\right)^{-1} .
$$

Clearly when $L=1$, meaning the function such that $L(e)=1$ for all edges $e$ in $X$, we have $\zeta(u, X, 1)=\zeta(u, X)$, our original Ihara zeta function. Then one would want to find a determinant formula for this weighted zeta function. We can find an Ihara determinant formula for integer weights by inflating the graph.

Definition 2.5. Given a graph $X$ with integer-valued weight function $L$, define the inflated graph $X_{L}$ in which each edge $e$ is replaced by an edge with $L(e)-1$ new degree 2 vertices.

Then clearly $\nu_{X}(C, L)=\nu_{X_{L}}(C, 1)$,where the 1 means again that $1(e)=1$, for all edges $e$. It follows that for integer-valued weights $L$, we have the identity relating the weighted zeta and the ordinary Ihara zeta:

$$
\zeta(u, X, L)=\zeta\left(u, X_{L}\right)
$$

and thus an Ihara determinant formula holds for this integer weighted graph zeta. It follows that $\zeta(u, X, L)^{-1}$ is a polynomial for integer valued weights $L$. For noninteger weights, we get a determinant formula for the general weighted Ihara zeta function in the next section.

Example. Suppose $Y=K_{5}$, the complete graph on 5 vertices. Let $L(e)=5$ for each of the 10 edges of $X$. Then $X=Y_{L}$ is the graph on the left in Figure 1. One sees easily that $\zeta\left(u^{5}, K_{5}\right)=\zeta(u, X)=\left(1-u^{10}\right)^{5}\left(1-3 u^{5}\right)\left(1-u^{5}\right)\left(1+u^{5}+3 u^{10}\right)$.

Kotani and Sunada [18] show that, for a non-circuit graph $X$, if $q+1$ is the maximum degree of $X$ and $p+1$ is the minimum degree of $X$, then every non-real pole $u$ of $\zeta_{X}(u)$ satisfies the inequality

$$
q^{-1 / 2} \leq|u| \leq p^{-1 / 2} .
$$


Moreover, they show that every pole $u$ of $\zeta_{X}(u)$ satisfies $R_{X} \leq|u| \leq 1$ and

$$
q^{-1} \leq R_{X} \leq p^{-1} \text {. }
$$

Next let us speak about Ramanujan graphs. These were defined for connected regular graphs by Lubotzky, Phillips, and Sarnak [22] and are of interest to computer scientists because the standard random walk on such graphs converges as fast as possible to uniform (at least in the non bipartite case) for a sequence of connected k-regular graphs such that the number of vertices approaches infinity. See Terras $[\mathbf{3 1}]$ and the references contained there. Other references for Ramanjujan graphs are: Fan Chung [4], Giuliana Davidoff, Peter Sarnak and Alain Valette [6], Alexander Lubotzky [20], and the paper of W.-C. Winnie Li in the volume of Hejhal et al [12].

First define two constants associated to the graph $X$.

DEFINITION 2.6.

$$
\begin{aligned}
& \rho_{X}=\max \left\{|\lambda| \mid \lambda \in \operatorname{spectrum}\left(A_{X}\right)\right\}, \\
& \rho_{X}^{\prime}=\max \left\{|\lambda|\left|\lambda \in \operatorname{spectrum}\left(A_{X}\right),\right| \lambda \mid \neq \rho_{X}\right\} .
\end{aligned}
$$

We will say that the naive Ramanujan inequality is

$$
\rho_{X}^{\prime} \leq 2 \sqrt{\rho_{X}-1}
$$

Lubotzky [21] has defined $X$ to be Ramanujan if

$$
\rho_{X}^{\prime} \leq \sigma_{X} .
$$

where $\sigma_{X}$ is the spectral radius of the adjacency operator on the universal covering tree of $X$. Both inequalities (2.7) and (2.8) reduce to the ususal definition of Ramanujan for connected regular graphs.

Hoory [13] has proved that if $\overline{d_{X}}$ denotes the average degree of the vertices of $X$, then

$$
2 \sqrt{\overline{d_{X}}-1} \leq \sigma_{X}
$$

Thus one has a criterion for a graph $X$ to be Ramanujan in Lubotzky's sense. It need only satisfy the Hoory inequality

$$
\rho_{X}^{\prime} \leq 2 \sqrt{\overline{d_{X}}-1}
$$

For a $(q+1)$-regular graph the standard change of variables $u=q^{-s}, s \in \mathbb{C}$, turns $\zeta(u, X)$ into a Dirichlet series which is zero- and pole-free for $\operatorname{Re}(s)>1$ and has a first order pole at $s=1$. In the new s-variable, there are functional equations relating the value of $f(s)=\zeta\left(q^{-s}, X\right)$ with $f(1-s)$ (see [28]). The RH (= Riemann hypothesis) says (e.g., see $[\mathbf{2 8}]$, p. 129) $\zeta_{X}(u)$ has no poles with $0<\operatorname{Re}(s)<1$ unless $\operatorname{Re}(s)=1 / 2$. D. Newland $[\mathbf{2 3}]$ has done computer experiments showing that for the pseudo-random regular graphs produced by Mathematica the spacings of the imaginary parts of the poles of $f(s)=\zeta\left(q^{-s}, X\right)$ appear to be those of the eigenvalues of a random real symmetric matrix ( $\mathrm{GOE}=$ Gaussian Orthogonal Ensemble) with a change of variables coming from the formula relating the poles of the Ihara zeta function and the eigenvalues of the adjacency matrix.

To develop the $\mathrm{RH}$ for irregular graphs, the natural change of variable is $u=R_{X}^{s}$ with $R_{X}$ from Definition 2.1. All poles of $\zeta_{X}(u)$ are then located in the "critical strip", $0 \leq \operatorname{Re}(s) \leq 1$ with poles at $s=0(u=1)$ and $s=1\left(u=R_{X}\right)$. From this point of view, it is natural to say that the Riemann hypothesis for $X$ should 
require that $\zeta_{X}(u)$ has no poles in the open strip $1 / 2<\operatorname{Re}(s)<1$. This is the graph theory $\mathrm{RH}$ below. After looking at examples, it seems that one rarely sees an Ihara zeta satisfying this $\mathrm{RH}$ so we weaken it to the weak graph theory $\mathrm{RH}$ below. The examples below show that in general one cannot expect a functional equation relating $f(s)=\zeta\left(R_{X}^{s}, X\right)$ and $f(1-s)$.

(Graph theory $\mathbf{R H}) \zeta_{X}(u)$ is pole free for

$$
R_{X}<|u|<\sqrt{R_{X}}
$$

(Weak graph theory $\mathbf{R H}) \zeta_{X}(u)$ is pole free for

$$
R_{X}<|u|<1 / \sqrt{q} \text {. }
$$

Note that we have examples (such as Examples 1 and 4 below) for which $R_{X}>$ $q^{-\frac{1}{2}}$ and in such cases the weak graph theory RH is true but vacuous. In [30] we give a longer discussion of the preceding 2 versions of the $\mathrm{RH}$ for graphs showing the connections with the versions for the Dedekind zeta function and the existence of Siegel zeros.

Sometimes number theorists state a modified GRH $=$ Generalized Riemann Hypothesis for the Dedekind zeta function and this just ignores all possible real zeros while only requiring the non-real zeros to be on the line $\operatorname{Re}(s)=\frac{1}{2}$. The graph theory analog of the modified weak GRH would just ignore the real poles and require that there are no non-real poles of $\zeta_{X}(u)$ in $R_{X}<|u|<q^{-1 / 2}$. But this is true for all graphs by a theorem in [18] quoted above in (2.5): if $\mu$ is a pole of $\zeta_{X}(u)$ and $|\mu|<q^{-1 / 2}$ then $\mu$ is real!

One may ask about the relations between the constants $\rho_{X}, \overline{d_{X}}, R_{X}$. One can show that

$$
\rho_{X} \geq \overline{d_{X}} .
$$

This is easily seen using the fact that $\rho_{X}$ is the maximum value of the Rayleigh quotient $\langle A f, f\rangle /\langle f, f\rangle$, while $\overline{d_{X}}$ is the value when $f$ is the vector all of whose entries are 1. In all the examples to date we see that $\rho_{X} \geq 1+\frac{1}{R_{X}} \geq \overline{d_{X}}$ but can only show that $\rho_{X} \geq \frac{p}{q}+\frac{1}{R_{X}}$.

Next we give some examples including answers to the questions: Do the spectra of the adjacency matrices satisfy the naive Ramanujan inequality (2.7) or the Hoory inequality (2.9)? Do the Ihara zeta functions for the graphs have the pole-free region (2.11) of the weak graph theory RH or the pole-free region (2.10) of the full graph theory RH?

Example 1. $X=$ the graph obtained from $K_{5}$ by adding 4 vertices to each edge as shown on the left in Figure 1.

We thank Adam O'Neill for his help with this example. For the graph $X$, we find that $\rho^{\prime} \approx 2.32771$ and $\left\{\rho, 1+1 / R, \overline{d_{X}}\right\} \approx\{2.39138,2.24573,2.22222\}$. It follows that this graph satisfies the naive Ramanujan inequality (2.7) but not the Hoory inequality (2.9). The picture on the right in Figure 1 shows stars for the poles not equal to -1 of $\zeta(u, X)$. Here

$$
\zeta(u, X)^{-1}=\zeta\left(u^{5}, K_{5}\right)^{-1}=\left(1-u^{10}\right)^{5}\left(1-3 u^{5}\right)\left(1-u^{5}\right)\left(1+u^{5}+3 u^{10}\right) .
$$

The circles in the picture on the right in Figure 1 are centered at the origin with radii $\left\{q^{-\frac{1}{2}}, R, R^{\frac{1}{2}}, p^{-\frac{1}{2}}\right\} \approx\{0.57735,0.802742,0.895958,1\}$. The zeta function satisfies the $\mathrm{RH}$ and thus the weak RH. However the weak RH is vacuous. 


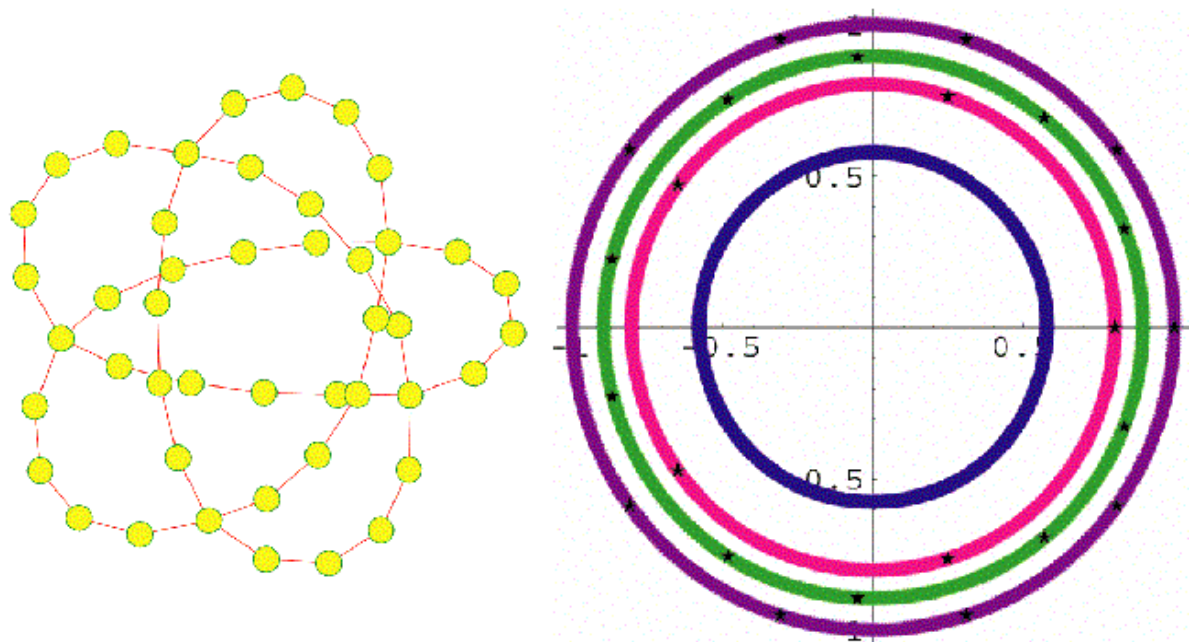

Figure 1 . On the left is the graph $X=Y_{5}$ obtained by adding 4 vertices to each edge of $Y=K_{5}$, the complete graph on 5 vertices. On the right the poles $(\neq-1)$ of the vertex Ihara zeta function of $X$ are shown by stars. The circles have centers at the origin and radii $\left\{q^{-\frac{1}{2}}, R, R^{\frac{1}{2}}, p^{-\frac{1}{2}}\right\}$.

\section{Example 2. Random graph with probability $1 / 2$ of an edge.}

See Figure 2 for the location of the poles not equal to \pm 1 of the vertex Ihara zeta function of a random graph produced by Mathematica with the command RandomGraph[100,1/2]. This means there are 100 vertices and the probability of an edge between any 2 vertices is $1 / 2$. The graph satisfies the Hoory inequality (2.9) and it is thus Ramanujan in Lubotzky's sense and satisfies the naive Ramanujan inequality (2.7). We find that $\rho^{\prime} \approx 10.3357$ and $\left\{\rho, 1+1 / R, \overline{d_{X}}\right\}$ $\approx\{48.5972,48.585,48$.$\} . The circles in Figure 2$ are centered at the origin and have radii given by $\left\{R, q^{-\frac{1}{2}}, R^{\frac{1}{2}}, p^{-\frac{1}{2}}\right\} \approx\{0.021015,0.129099,0.144966,0.174078\}$. The poles of the zeta function satisfy the weak RH but not the RH. See Skiena [27] for more information on the model that Mathematica uses to produce random graphs.

\section{Example 3. Torus minus some edges.}

From the torus graph $T$ which is the product of a 3 -cycle and a 10-cycle, we delete 7 edges to obtain a graph we will call $N$ which is on the left in Figure 3. The spectrum of the adjacency matrix of $N$ satisfies neither the Hoory inequality (2.9) nor the naive Ramanujan inequality (2.7). We find that $\{\rho, 1+1 / R, d\} \approx$ $\{3.60791,3.58188,3.53333\}$, and $\rho^{\prime} \approx 3.39492$. The right hand side of Figure 3 shows the poles of the vertex zeta for $N$ as stars. The circles have center at the origin and radii $\left\{R, q^{-\frac{1}{2}}, R^{\frac{1}{2}}, p^{-\frac{1}{2}}\right\} \approx\{0.387315,0.57735,0.622347,0.707107\}$. The zeta poles satisfy neither the graph theory weak $\mathrm{RH}$ nor the $\mathrm{RH}$. 


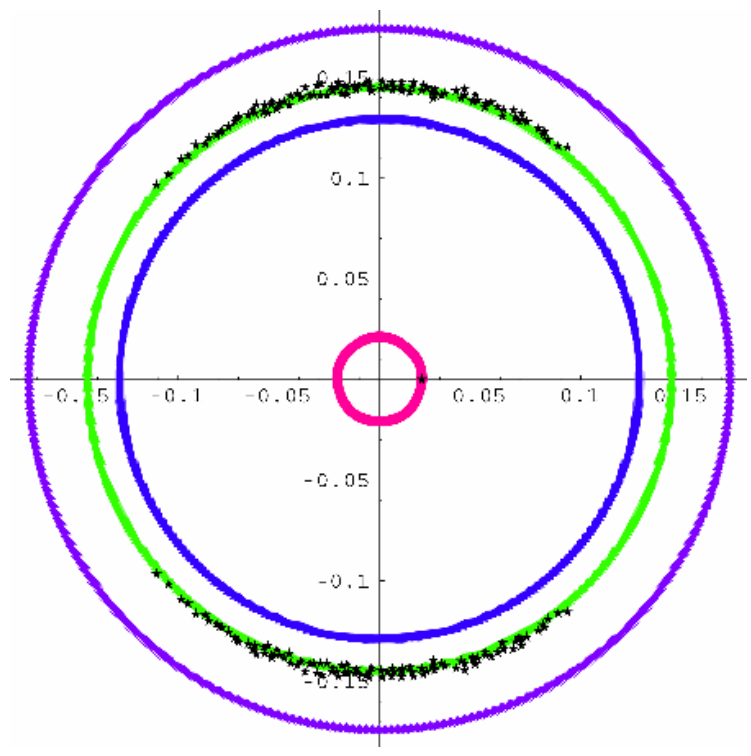

Figure 2. The poles $(\neq \pm 1)$ of the vertex Ihara zeta function for a random graph produced by Mathematica with the command RandomGraph $\left[100, \frac{1}{2}\right]$. The circles have centers at the origin and radii $\left\{R, q^{-\frac{1}{2}}, R^{\frac{1}{2}}, p^{-\frac{1}{2}}\right\}$.
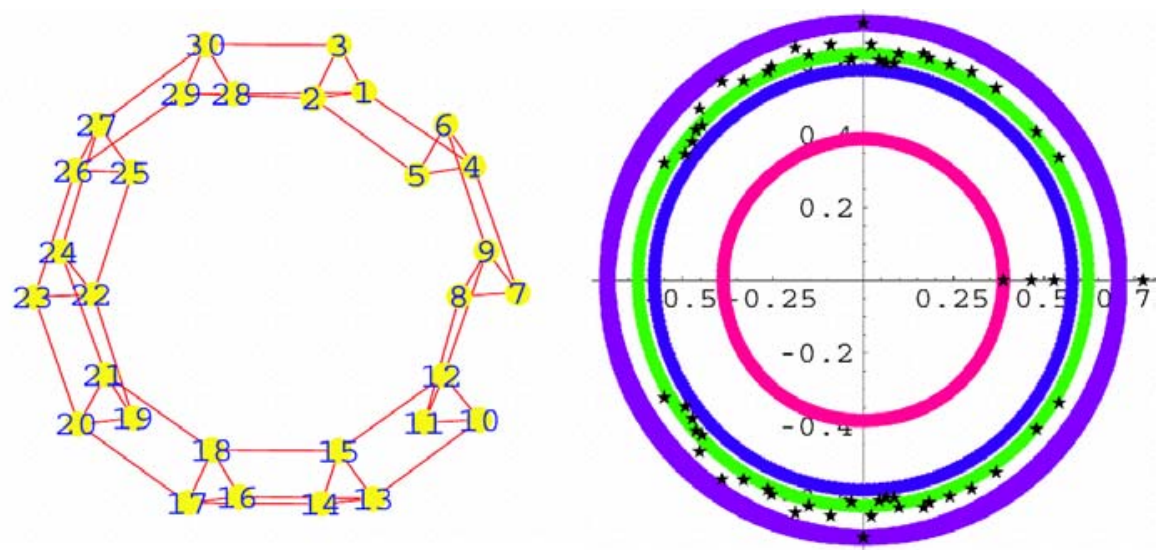

Figure 3 . The graph $N$ on the left results from deleting 7 edges from the product of a 3 -cycle and a 10-cycle. In the picture on the right, stars indicate the poles $(\neq \pm 1)$ of the vertex Ihara zeta function of $N$. The circles are centered at the origin with radii $\left\{R, q^{-1 / 2}, R^{1 / 2}, p^{-1 / 2}\right\}$. 

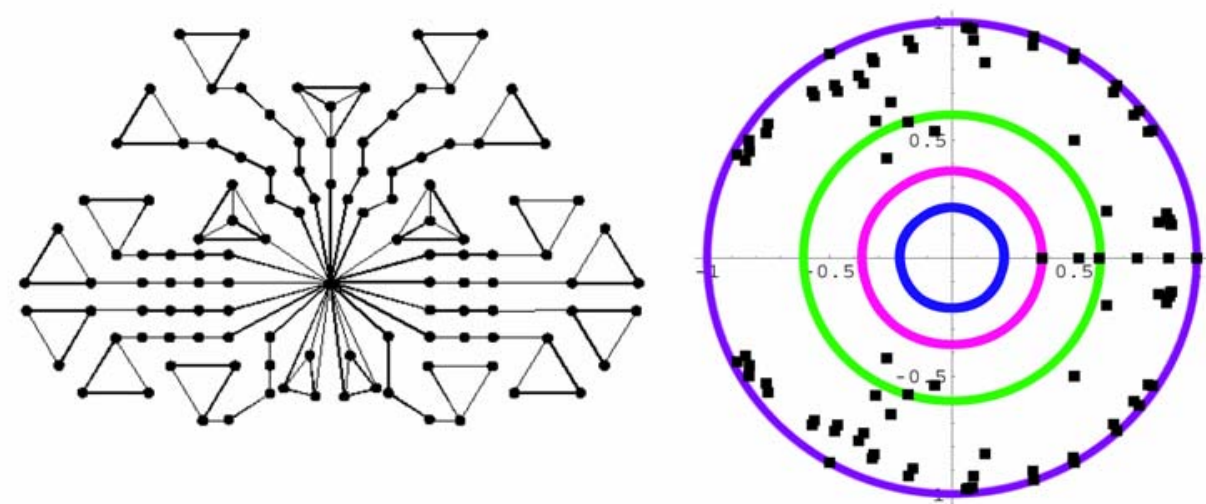

Figure 4 . On the left is the e-graph $E$ of Example 4 with the property that $R^{-1}$ approximates $e$ to 7 digits. On the right the poles $(\neq-1)$ of the vertex Ihara zeta function of $E$ are indicated by boxes. The circles have centers at the origin and radii $\left\{q^{-\frac{1}{2}}, R, R^{\frac{1}{2}}, p^{-\frac{1}{2}}\right\}$.

Example 4. The e-graph $E$ found by M. Horton $[\mathbf{1 5}]$ has the property that $1 / R \approx 2.7182819$. It is pictured on the left in Figure 4 .

We find that $\{\rho, 1+1 / R, d\} \approx\{5.26243,3.71828,2.47863\}$. The spectrum of the adjacency matrix of $E$ satisfies neither the Hoory inequality (2.9) nor the naive Ramanujan inequality (2.7). It is open whether it is Ramanujan in Lubotzky's sense. The right hand side of Figure 4 shows the poles $(\neq-1)$ of the vertex zeta function of $E$ as boxes. The circles have center at the origin and radii $\left\{q^{-\frac{1}{2}}, R, R^{\frac{1}{2}}, p^{-\frac{1}{2}}\right\} \approx\{0.213201,0.367879,0.606531,1\}$. The weak $\mathrm{RH}$ is vacuous and thus true, while the $\mathrm{RH}$ is false.

Next we intend to prove the graph theory prime number theorem. First we need some definitions.

Definition 2.7. The closed path counting function $N_{m}$ is the number of closed paths $C$ of length $m$ in $X$ without backtracking or tails.

DeFinition 2.8. The prime counting function is

$$
\pi(n)=\#\{[C]=\text { prime in } X \text { with length of } C \text { equal to } n\} .
$$

Note that in $\pi(n)$ we count equivalence classes of paths and not paths.

Definition 2.9. The g.c.d. of the prime path lengths in $X$ is

$$
\Delta_{X}=\text { g.c.d. }\{\nu(P) \mid[P]=\text { a prime in } X\} .
$$

Theorem 2.10. (Graph Prime Number Theorem) Suppose that $R_{X}$ is as in Definition 2.1. Then for a connected graph $X$ with $\Delta_{X}=1$ we have

$$
\pi(m) \sim \frac{R_{X}^{-m}}{m}, \quad \text { as } \quad m \rightarrow \infty .
$$


If $\Delta_{X}>1$, then $\pi(m)=0$ unless $\Delta_{X}$ divides $m$. If $\Delta_{X}$ divides $m$, we have

$$
\pi(m) \sim \Delta_{X} \frac{R_{X}^{-m}}{m}, \quad \text { as } \quad m \rightarrow \infty .
$$

Proof. Recall formula (2.2) defining the Ihara zeta function. Then

$$
\begin{aligned}
u \frac{d}{d u} \log \zeta(u, X) & =-u \frac{d}{d u} \sum_{[P]} \log \left(1-u^{\nu(P)}\right) \\
& =\sum_{[P]} \sum_{j \geq 1} \frac{1}{j} u \frac{d}{d u} u^{\nu(P) j}=\sum_{[P]} \sum_{j \geq 1} \frac{\nu(P) j}{j} u^{\nu(P) j} .
\end{aligned}
$$

The outer sum is over equivalence classes $[P]$ of prime closed paths with no backtracking or tails. Sum over all primes $[P]$ with $\nu(P)=d$. Now there are $d$ elements in $[P]$. So we can drop the [ ] and obtain a sum over all closed paths $C=P^{j} \quad$ without backtracking or tails. This gives

$$
u \frac{d}{d u} \log \zeta(u, X)=\sum_{m \geq 1} N_{m} u^{m}
$$

Next observe that the defining formula for the Ihara zeta function can be written as

$$
\zeta(u, X)=\prod_{n \geq 1}\left(1-u^{n}\right)^{\pi(n)}
$$

Then

$$
u \frac{d}{d u} \log \zeta(u, X)=\sum_{n \geq 1} \frac{n \pi(n) u^{n}}{1-u^{n}}=\sum_{m \geq 1} \sum_{d \mid m} d \pi(d) u^{m} .
$$

Here the inner sum is over all positive divisors of $m$. Thus we obtain the following relation between the functions on $\mathbb{Z}^{+}$which we have called $N_{m}$ and $\pi(n)$.

$$
N_{m}=\sum_{d \mid m} d \pi(d)
$$

This sort of relation occurs frequently in number theory and combinatorics. It is inverted using the Möbius function $\mu(n)$ defined by

$$
\mu(n)=\left\{\begin{array}{ccc}
1 & n=1 \\
(-1)^{r} & n=p_{1} \cdots p_{r}, & \text { for distinct primes } p_{i} \\
0 & \text { otherwise. }
\end{array}\right.
$$

Then by the Möbius inversion formula

$$
\pi(m)=\frac{1}{m} \sum_{d \mid m} \mu\left(\frac{m}{d}\right) N_{d}
$$

Next we look at formula (2.4) and apply our differential operator after using the Schur upper triangularization of $W_{1}$ (see Horn and Johnson [14]). This gives

$$
u \frac{d}{d u} \log \zeta(u, X)=-\sum_{\lambda \in \operatorname{Spec}\left(W_{1}\right)} \log (1-\lambda u)=\sum_{\lambda \in \operatorname{Spec}\left(W_{1}\right)} \sum_{n \geq 1} \frac{1}{n}(\lambda u)^{n} .
$$

It follows that

$$
N_{m}=\sum_{\lambda \in \operatorname{Spec}\left(W_{1}\right)} \frac{\lambda^{m}}{m}
$$


The dominant terms in this last sum are those coming from $\lambda \in \operatorname{Spec}\left(W_{1}\right)$ such that $|\lambda|=R^{-1}$. By Kotani and Sunada [18], Theorem 1.4, which is proved using the Perron-Frobenius theorem (see [14]), the largest absolute value of an eigenvalue $\lambda$ occurs $\Delta_{X}$ times with these eigenvalues having the form $e^{2 \pi i a / \Delta_{X}} R$, where $a=1, \ldots, \Delta_{X}$. Using the orthogonality relations for exponential sums (see $[\mathbf{3 1}]$ ) which are basic to the theory of the finite Fourier transform, we see that

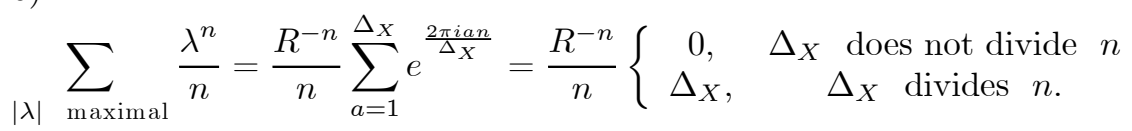

The graph prime number theorem follows from formulas (2.14), (2.15), and (2.16).

The vertex Ihara zeta function can be used to determine the rank of the fundamental group, for it is the order of the pole of the Ihara zeta function at $u=1$. The complexity $\kappa_{X}$ of a graph is defined to be the number of spanning trees in $X$. One can use the matrix-tree theorem (see Biggs $[\mathbf{3}]$ ) to prove that

$$
\left(\frac{1}{\zeta_{V}(u, X)}\right)^{(r)}(1)=r !(-1)^{r+1} 2^{r}(r-1) \kappa_{X} .
$$

Here the superscript (r) means the rth derivative. This result is an exercise on the last page of Terras [31], where some hints are given. It is an analog of the formula for the Dedekind zeta function of a number field at 0 - a formula involving the class number and the regulator of the number field. See Lang $[\mathbf{1 9}]$.

\section{Edge Zeta Functions}

Here we will give our version of the Bass proof of Ihara's determinant formula. This implies that there is a determinant formula for the vertex zeta function of weighted graphs even if the weights are not integers. In an example we will discuss what deleting an edge of a graph (fission) does to the edge zeta function.

Definition 3.1. The edge matrix $W$ for graph $X$ is a $2 m \times 2 m$ matrix with $a, b$ entry corresponding to the oriented edges $a$ and $b$. This $a, b$ entry is the complex variable $w_{a b}$ if edge $a$ feeds into edge $b$ and $b \neq a^{-1}$ and the $a, b$ entry is 0 otherwise.

Definition 3.2. Given a path $C$ in $X$, which is written as a product of oriented edges $C=a_{1} a_{2} \cdots a_{s}$, the edge norm of $C$ is

$$
N_{E}(C)=w_{a_{1} a_{2}} w_{a_{2} a_{3}} \cdots w_{a_{s-1} a_{s}} w_{a_{s} a_{1}} .
$$

The edge Ihara zeta function is

$$
\zeta_{E}(W, X)=\prod_{[P]}\left(1-N_{E}(P)\right)^{-1},
$$

where the product is over primes in $X$.

Clearly if you set all non-zero variables in $W$ equal to $u$, the edge zeta function specializes to the vertex zeta function; i.e,

$$
\left.\zeta_{E}(W, X)\right|_{0 \neq w_{a b}=u}=\zeta_{V}(u, X) .
$$


If $X$ is a weighted graph with weight function $L$, and you specialize the non-zero variables $w_{a b}=u^{(L(a)+L(b)) / 2}$, you get the weighted Ihara zeta function. Or you could specialize $w_{a b}=u^{L(a)}$.

If you cut an edge of a graph, you can compute the edge zeta for the new graph with one less edge by setting all variables equal to 0 if the cut edge or its inverse appear in a subscript.

The edge zeta again has a determinant formula and is the reciprocal of a polynomial in the $w_{a b}$ variables. This is the following theorem whose proof should be compared with the Bowen Lanford proof of a similar theorem for dynamical zeta functions. See Ruelle [25] or Bedford, Keane, and Series [2].

Theorem 3.3. (Determinant Formula for the Edge Zeta).

$$
\zeta_{E}(W, X)=\operatorname{det}(I-W)^{-1} .
$$

Proof. (Sketch) First note that

$$
-\log \zeta_{E}(W, X)=\sum_{[P]} \sum_{j \geq 1} \frac{1}{j} N_{E}(P)^{j} .
$$

Let $L=\sum_{i, j} w_{i j} \frac{\partial}{\partial w_{i j}}$ be the Euler operator. Then, since there are $\nu(P)$ elements in $[P]$, we have

$$
-L \log \zeta_{E}(W, X)=\sum_{\substack{m \geq 1 \\ j \geq 1}} \frac{1}{j m} \sum_{\substack{P \\ \nu(P)=m}} L\left(N_{E}(P)^{j}\right) .
$$

Now $\nu(P)=m$ implies $L\left(N_{E}(P)^{j}\right)=j m N_{E}(P)^{j}$. It follows that

$$
-L \log \zeta_{E}(W, X)=\sum_{C} N_{E}(C) .
$$

Here the sum is the path $C$ need not be a prime path, but it is still closed without backtracking or tail. Finally we see that

$$
-L \log \zeta_{E}(W, X)=\sum_{m \geq 1} \operatorname{Tr}\left(W^{m}\right) .
$$

Next you need to do an exercise in matrix calculus and show that the right hand side of the preceding formula is $L \log \operatorname{det}(I-W)^{-1}$ This proves $L(\log ($ determinant formula $))$. To finish the proof, observe that both sides are 1 when all the $w_{a b}$ are 0 .

By formula (3.1), we have the following Corollary, since specializing all the non-zero variables in $W$ to be $u$, yields the matrix $u W_{1}$.

Corollary 1. $\zeta_{V}(u, X)=\operatorname{det}\left(I-u W_{1}\right)^{-1}$. 


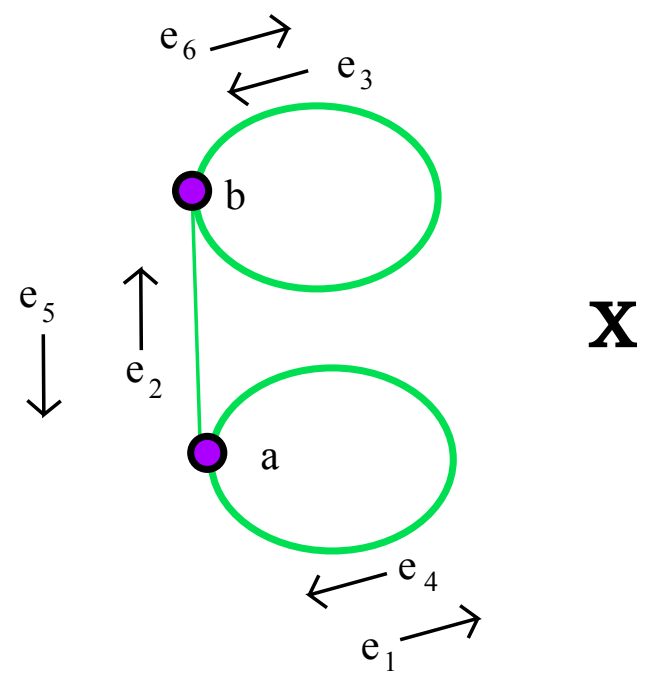

Figure 5. The dumbell graph

\section{Example. Dumbbell Graph.}

Figure 5 shows the labeled picture of the dumbbell graph $X$. For this graph we find that $\zeta_{E}(W, X)^{-1}=$

$$
\operatorname{det}\left(\begin{array}{cccccc}
w_{11}-1 & w_{12} & 0 & 0 & 0 & 0 \\
0 & -1 & w_{23} & 0 & 0 & w_{26} \\
0 & 0 & w_{33}-1 & 0 & w_{35} & 0 \\
0 & w_{42} & 0 & w_{44}-1 & 0 & 0 \\
w_{51} & 0 & 0 & w_{54} & -1 & 0 \\
0 & 0 & 0 & 0 & w_{65} & w_{66}-1
\end{array}\right) .
$$

Note that if we cut the vertical edges which are edges $e_{2}$ and $e_{5}$, we should specialize all the variables with 2 or 5 in them to be 0 . This yields the edge zeta function of the subgraph with the vertical edge removed, and incidentally diagonalizes the matrix $W$. We call this "fission". The edge zeta is particularly suited to keeping track of such fission.

Next we give a version of Bass's proof of the Ihara determinant formula using the preceding theorem. In what follows, $n$ is the number of vertices of $X$ and $m$ is the number of unoriented edges of $X$.

First define some matrices. Set $J=\left(\begin{array}{cc}0 & I_{m} \\ I_{m} & 0\end{array}\right)$. Then define the $n \times 2 m$ start matrix $S$ and the $n \times 2 m$ terminal matrix $T$ by setting

$$
s_{v e}=\left\{\begin{array}{l}
1, \quad \text { if } v \text { is starting vertex of edge } e, \\
0, \\
\text { otherwise }
\end{array}\right.
$$

and

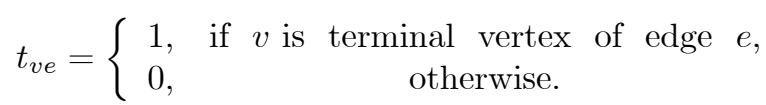


Proposition 1. (Some Matrix Identities) Using the preceding definitions, the following formulas hold. We write ${ }^{t} M$ for the transpose of the matrix $M$.

1) $S J=T, \quad T J=S$.

2) If $A$ is the adjacency matrix of $X$ and $Q+I_{n}$ is the diagonal matrix whose jth diagonal entry is the degree of the jth vertex of $X$, then $A=S{ }^{t} T$, and $Q+I_{n}=$ $S^{t} S=T{ }^{t} T$.

3) The matrix $W_{1}$ from Definition 2.2 satisfies $W_{1}+J={ }^{t} T S$.

ProOF. 1) comes from the fact that the starting (terminal) vertex of edge $e_{j}$ is the terminal (starting) vertex of edge $e_{j+|E|}$, according to our edge numbering system from formula (2.1).

2) Consider

$$
\left(S^{t} T\right)_{a, b}=\sum_{e} s_{a e} t_{b e}
$$

The right hand side is the number of edges $e$ such that $a$ is the initial vertex and $b$ is the terminal vertex of $e$, which is the $a, b$ entry of $A$. Similar arguments prove the second formula.

3) We have

$$
\left({ }^{t} T S\right)_{e f}=\sum_{v} t_{v e} s_{v f}
$$

The sum is 1 iff edge $e$ feeds into edge $f$, even if $f=e^{-1}$.

\section{Bass's Proof of the Ihara Determinant Formula (2.3).}

PROOF. In the following identity all matrices are $(n+2 m) \times(n+2 m)$, where the 1st block is $n \times n$, if $n$ is the number of vertices of $X$ and $m$ is the number of unoriented edges of $X$. Use the preceding proposition to see that

$$
\begin{aligned}
& \left(\begin{array}{cc}
I_{n} & 0 \\
{ }^{t} T & I_{2 m}
\end{array}\right)\left(\begin{array}{cc}
I_{n}\left(1-u^{2}\right) & S u \\
0 & I_{2 m}-W_{1} u
\end{array}\right) \\
= & \left(\begin{array}{cc}
I_{n}-A u+Q u^{2} & S u \\
0 & I_{2 m}+J u
\end{array}\right)\left(\begin{array}{cc}
I_{n} & 0 \\
{ }^{t} T-{ }^{t} S u & I_{2 m}
\end{array}\right) .
\end{aligned}
$$

Take determinants to obtain

$$
\left(1-u^{2}\right)^{n} \operatorname{det}\left(I-W_{1} u\right)=\operatorname{det}\left(I_{n}-A u+Q u^{2}\right) \operatorname{det}\left(I_{2 m}+J u\right) .
$$

To finish the proof of formula (2.3), observe that

$$
I+J u=\left(\begin{array}{cc}
I & I u \\
I u & I
\end{array}\right)
$$

implies

$$
\left(\begin{array}{cc}
I & 0 \\
-I u & I
\end{array}\right)(I+J u)=\left(\begin{array}{cc}
I & I u \\
0 & I\left(1-u^{2}\right)
\end{array}\right) .
$$

Thus $\operatorname{det}(I+J u)=\left(1-u^{2}\right)^{m}$. Since $r-1=m-n$, for a connected graph, the Ihara formula $(2.3)$ follows. 


\section{Path Zeta Functions}

Here we look at a zeta function invented by Stark. It has several advantages over the edge zeta. It can be used to compute the edge zeta with smaller determinants. It gives the edge zeta for a graph in which an edge has been fused; i.e., shrunk to one vertex.

First recall that the fundamental group of $X$ can be identified with the group generated by the edges left out of a spanning tree $T$ of $X$. We call the oriented versions of these edges left out of the spanning tree $T$ (or "cut" edges of T) (and their inverses)

$$
e_{1}, \ldots, e_{r}, e_{1}^{-1}, \ldots, e_{r}^{-1} \text {. }
$$

Call the remaining (oriented) edges (and their inverses) in the spanning tree $T$

$$
t_{1}, \ldots, t_{u}, t_{1}^{-1}, \ldots, t_{u}^{-1} .
$$

Definition 4.1. The $2 r \times 2 r$ path matrix $Z$ has $i j$ entry given by the complex variable $z_{i j}$ if $e_{i} \neq e_{j}^{-1}$ and by 0 if $e_{i}=e_{j}^{-1}$.

Note that the path matrix $Z$ has only one zero entry in each row unlike the edge matrix $W$ from Definition 3.1 which is rather sparse.

Next we imitate the definition of the edge zeta function. So we define the path norm for a prime path $C=a_{1} \cdots a_{s}$, where $a_{i} \in\left\{e_{1}^{ \pm 1}, \ldots, e_{s}^{ \pm 1}\right\}$ as

$$
N_{F}(C)=z_{a_{1} a_{2}} \cdots z_{a_{s-1} a_{s}} z_{a_{s} a_{1}} .
$$

Then the path zeta is defined to be

$$
\zeta_{F}(Z, X)=\prod_{[P]}\left(1-N_{F}(P)\right)^{-1} .
$$

where the product is over primes $[P]$.

The determinant formula for the path zeta is proved similarly to that for the edge zeta and it says

$$
\zeta_{F}(Z, X)^{-1}=\operatorname{det}(I-Z) .
$$

Next we want to give a way to get the edge zeta out of the path zeta. To do this requires a procedure called specializing the path matrix to the edge matrix. Use the notation above for the edges left out of the spanning tree $T$ and the edges of $T$. A prime cycle $P$ is first written as a product of generators of the fundamental group and then as a product of actual edges $e_{i}$ and $t_{k}$. Do this by inserting $t_{k_{1}} \cdots t_{k_{s}}$ which is the unique non backtracking path on $T$ joining the terminal vertex of $e_{i}$ and the starting vertex of $e_{j}$ if $e_{i}$ and $e_{j}$ are successive cut edges in $P$. Now specialize the path matrix $Z$ to $Z(W)$ with entries

$$
z_{i j}=w_{e_{i} t_{k_{1}}} w_{t_{k_{1}} t_{k_{2}}} \cdots w_{t_{k_{n-1}} t_{k_{n}}} w_{t_{k_{n}} e_{j}} .
$$

Then the path zeta function at $\mathrm{Z}(\mathrm{W})$ specializes to the edge zeta function

$$
\zeta_{F}(Z(W), X)=\zeta_{E}(W, X) .
$$

M. Horton $[\mathbf{1 5}]$ has a Mathematica program to do the specialization in formula (4.1). 


\section{Example. The Dumbbell Again.}

Recall that the edge zeta of the dumbbell graph of Figure 5 was evaluated by a $6 \times 6$ determinant. The path zeta requires a $4 \times 4$ determinant. Take the spanning tree to be the vertical edge. That is really the only choice here. One finds using the determinant formula for the path zeta and the specialization of the path to edge zeta:

$$
\zeta_{E}(W, X)^{-1}=
$$

$$
\operatorname{det}\left(\begin{array}{cccc}
w_{11}-1 & w_{12} w_{23} & 0 & w_{12} w_{26} \\
w_{35} w_{51} & w_{33}-1 & w_{35} w_{54} & 0 \\
0 & w_{42} w_{23} & w_{44}-1 & w_{42} w_{26} \\
w_{65} w_{51} & 0 & w_{65} w_{54} & w_{66}-1
\end{array}\right)
$$

If we shrink the vertical edge to a point (which we call "fusion"), the edge zeta of the new graph is obtained by replacing any $w_{x 2} w_{2 y}$ (for $\left.x, y=1,3,4,6\right)$ which appear in formula (4.2) by $w_{x y}$ and any $w_{x 5} w_{5 y}$ (for $\left.x, y=1,3,4,6\right)$ by $w_{x y}$. This gives the zeta function of the new graph obtained from the dumbbell, by fusing the vertical edge.

\section{References}

[1] H. Bass, The Ihara-Selberg zeta function of a tree lattice, Internatl. J. Math., 3 (1992), 717-797.

[2] T. Bedford, M. Keane, and C. Series, (Eds), Ergodic Theory, Symbolic Dynamics, and Hyperbolic Spaces, Oxford U. Press, Oxford, 1991.

[3] N. Biggs, Algebraic Graph Theory, Cambridge Univ. Press, London, 1974.

[4] F. R. K. Chung, Spectral Graph Theory, CBMS Regional Conf. Series in Math., No. 92, Amer. Math. Soc., Providence, 1996,

[5] B. Clair and S. Mokhtari-Sharghi, Zeta functions of discrete groups acting on trees. J. Algebra, 237 (2001), 591-620.

[6] G. Davidoff, P. Sarnak and A. Valette, Elementary Number Theory, Group Theory and Ramanujan Graphs, Cambridge U. Press, Cambridge, 2003.

[7] H. M. Edwards, Riemann's Zeta Function, Academic, N.Y., 1974.

[8] J. Elstrodt, Die Selbergsche Spurformel für kompakte Riemannsche Flächen, Jber. d. Dt. Math.-Verein., 83 (1981), 45-77.

[9] R. I. Grigorchuk and A. Zuk, The Ihara zeta function of infinite graphs, the KNS spectral measure and integrable maps, in Random walks and geometry, Walter de Gruyter, Berlin, 2004, pages 141-180.

[10] K. Hashimoto, Zeta functions of finite graphs and representations of p-adic groups, $A d v$. Studies in Pure Math., Vol. 15, Academic Press, N.Y., 1989, pp. 211-280.

[11] D. A. Hejhal, The Selberg trace formula and the Riemann zeta function, Duke Math. J., 43 (1976), 441-482.

[12] D. A. Hejhal, J. Friedman, M. Gutzwiller, A. Odlyzko (Eds.), Emerging Applications of Number Theory, Inst. for Math and its Applics. Vol. 109, Springer-Verlag, N.Y., 1999.

[13] S. Hoory, A lower bound on the spectral radius of the universal cover of a graph, J. of Combinatorial Theory, Series B, 93 (2005), 33-43.

[14] R. A. Horn and C. R. Johnson, Topics in Matrix Analysis, Cambridge U. Press, Cambridge, 1991.

[15] M. Horton, Ihara Zeta Functions of Irregular Graphs, Ph.D. Thesis, U.C.S.D., 2006.

[16] N. Katz and P. Sarnak, Zeros of zeta functions and symmetry, Bull. Amer. Math. Soc. (N.S.) 36 (1999), no. 1, 1-26.

[17] N. Katz and P. Sarnak, Random Matrices, Frobenius Eigenvalues and Monodromy, Amer. Math. Soc., Providence, RI, 1999.

[18] M. Kotani and T. Sunada, Zeta functions of finite graphs, J. Math. Sci. Univ. Tokyo, 7 (2000), 7-25.

[19] S. Lang, Algebraic Number Theory, Addison-Wesley, Reading, Mass., 1968. 
[20] A. Lubotzky, Discrete Groups, Expanding Graphs and Invariant Measures, Birkhäuser, Basel, 1994.

[21] A. Lubotzky, Cayley graphs: eigenvalues, expanders and random walks, in Surveys in Combinatorics, London Math. Soc. Lecture Notes, 218, Cambridge U. Press, Cambridge, 1995, pp. $155-189$.

[22] A. Lubotzky, R. Phillips and P. Sarnak, Ramanujan graphs, Combinatorica, 8 (1988), 261277.

[23] D. Newland, Kernels in the Selberg Trace Formula on the k-regular tree and Zeros of the Ihara Zeta Function, Ph.D. Thesis, U.C.S.D., 2005.

[24] M. Rosen, Number Theory in Function Fields, Springer-Verlag, N.Y., 2002.

[25] D. Ruelle, Dynamical Zeta Functions for Piecewise Monotone Maps of the Interval, American Math. Soc., Providence, 1994.

[26] A. Selberg, Collected Papers, Vol. I, Springer-Verlag, N.Y., 1989.

[27] S. Skiena, Implementing Discrete Mathematics: Combinatorics and Graph Theory with Mathematica, Addison-Wesley, Redwood City, CA, 1990.

[28] H. M. Stark and A. A. Terras, Zeta Functions of Finite Graphs and Coverings, Advances in Math., 121 (1996), 124-165.

[29] H. M. Stark and A. A. Terras, Zeta Functions of Finite Graphs and Coverings, II, Advances in Math., 154 (2000), 132-195.

[30] H. M. Stark and A. A. Terras, Zeta Functions of Finite Graphs and Coverings, III, preprint.

[31] A. Terras, Fourier Analysis on Finite Groups and Applications, Cambridge Univ. Press, Cambridge, U.K., 1999.

[32] A. Terras, Harmonic Analysis on Symmetric Spaces and Applications, I,II, Springer-Verlag, N.Y., 1985, 1988

[33] A. B. Venkov and A. M. Nikitin, The Selberg trace formula, Ramanujan graphs and some problems of mathematical physics, Petersburg Math. J., 5 (1994), 419-484.

Math. Dept., U.C.S.D., 9500 Gilman Drive, La Jolla, CA 92093-0112,

Current address: Math. Dept., U.C.S.D., 9500 Gilman Drive, La Jolla, CA 92093-0112

Math. Dept., U.C.S.D., 9500 Gilman Drive, La Jolla, CA 92093-0112

$U R L:$ http://www. math.ucsd.edu/ aterras 